\title{
AN LPV POLE-PLACEMENT APPROACH TO FRICTION COMPENSATION AS AN FTC PROBLEM
}

\author{
RON J. PATTON*, LEJUn CHEN* , SuPAT KLINKHIEO ** \\ * Department of Engineering \\ University of Hull, Cottingham, Hull, HU6 7RX, UK \\ e-mail: r.j.patton@hull.ac.uk \\ ${ }^{* *}$ Synchrotron Light Research Institute (SLRI) \\ P.O. Box 93, Nakhon-Ratchasima, 30000, Thailand
}

\begin{abstract}
The concept of combining robust fault estimation within a controller system to achieve active Fault Tolerant Control (FTC) has been the subject of considerable interest in the recent literature. The current study is motivated by the need to develop model-based FTC schemes for systems that have no unique equilibria and are therefore difficult to linearise. Linear Parameter Varying (LPV) strategies are well suited to model-based control and fault estimation for such systems. This contribution involves pole-placement within suitable LMI regions, guaranteeing both stability and performance of a multi-fault LPV estimator employed within an FTC structure. The proposed design strategy is illustrated using a nonlinear two-link manipulator system with friction forces acting simultaneously at each joint. The friction forces, regarded as a special case of actuator faults, are estimated and their effect is compensated within a polytope controller system, yielding a robust form of active FTC that is easy to apply to real robot systems.
\end{abstract}

Keywords: friction, linear parameter varying, fault detection and diagnosis, linear matrix inequality, pole-placement.

\section{Introduction}

Friction phenomena are widely encountered in physical (mechanical/mechatronic) systems and the problem of control of systems with friction presents interesting challenges. In recent years there has grown a substantial literature on this subject (Armstrong-Hélouvry et al., 1994; Olsson et al., 1998; Bona and Indri, 2005). From a control point of view, friction compensation strategies that require a detailed model of the friction characteristics have limitations arising from nonsmooth nonlinearity and the fact that friction modelling remains an imprecise subject, thereby resulting in a robustness limitation.

The challenge of developing FTC schemes for systems with friction is even greater, particularly when the friction effects can mask the presence of other unwanted (fault) phenomena. Friction forces degrade the closedsystem performance and the concept of compensating or removing the friction effects in a closed-loop system can be viewed as an FTC problem. In the general case the friction forces in a control system can be considered to act as system component faults, or as actuator or sensor faults. The idea of regarding friction forces as fault effects was first considered by Patton et al. (2010) in a study describing the use of sliding mode observer theory as a robust method of friction force estimation within an adaptive FTC fault compensation system, applied to an inverted pendulum system. Patton and Klinkhieo (2010) describe the concept of LPV fault estimation within an FTC fault compensation framework, applied to a two-joint manipulator system with simple perturbation faults. The current paper extends the theoretical approach of Patton and Klinkhieo (2010) by (a) providing a proof for the LPV estimator stability via pole-placement design in an LMI region, (b) including the use of an LPV stabilizing controller, and (c) introducing simultaneously acting friction forces based on the manipulator model used by Patton and Klinkhieo (2010).

The LPV modelling methodology has been widely adopted in control system design in recent years, especially in relation to vehicle and aerospace control ( $\mathrm{Wu}$, 2001; Ganguli et al., 2002). Fault Detection and Diagnosis (FDD) methods (including fault estimation) and FTC studies based on the LPV concept have also been developed in a number of works (Bokor and Balas, 2004; Henry 
and Zolghadri, 2005, Casavola et al. 2007; 2008; Weng et al., 2008). However, the inclusion of a pole-placement approach to guarantee the performance and stability requirements of a multivariable LPV estimator is a new contribution.

By using the LPV approach, the time-varying terms of a nonlinear system are parameterised via smooth measurements of suitable parameters. Hence, the nonlinear dynamics are modelled as a system with a time-varying linear structure (dependent on the measured parameters). For control applications the LPV approach provides a strategy for robust control and facilitates direct application of classical control methods. The LPV approach to control also provides a starting framework for FTC in terms of a baseline controller for the time-varying system.

LPV-based approaches to FDD can be divided into two categories: (i) a linear-fractional-representationformalism-based approach and (ii) a polytopic-formalismbased approach. This paper adopts the latter approach to design a robust multivariable LPV fault estimator for a nonlinear system with no unique equilibria.

The time-varying model system is represented by an LPV polytope structure. The system is controlled at each vertex via efficient interior-point algorithms and LMIs, used within an $H_{\infty}$ framework and based on the well known formalism of Apkarian et al. (1995). Using the same framework, an LMI-based LPV pole-placement strategy is developed from the time-varying measured parameters. The LPV estimator is applicable to systems that have no unique equilibria. It is shown that the multidimensional LPV fault estimator with pole-placement can be determined efficiently with the solution of a set of LMIs, and the friction forces are constructed effectively and used in a compensation mechanism in each control signal. The LMI-based pole-placement design is utilised to guarantee the performance and stability of the estimator. The concept of fault effect factors, first defined by Chen et al. (1999), is outlined as a mechanism for achieving an active FTC scheme in which the nominal performance can be recovered.

Being different from simple fault detection, wherein the sensitivity of the fault is necessary, fault estimation requires the achievement of the fault magnitude, error steady state error. Therefore, the requirement of maximizing fault sensitivity can be transformed into that of minimizing the fault error. In other words, the $H_{\infty} / H_{-}$ fault residual generator of Hou and Patton (1996) can be viewed as an $H_{\infty}$ fault estimator. The literature on the development of the optimization-based method is well summarized by Marcos et al. (2005).

A nonlinear two-link manipulator system (adopted from the work of Patton and Klinkhieo (2010)) is a good choice of an example to demonstrate the effectiveness of fault estimation and the FTC scheme as it has no unique equilibria, requiring the use of the LPV approach. Fric- tion forces are simulated using the well-known Stribeck friction model and combined with the manipulator system as disturbance inputs. The estimated friction forces show good correspondence with their real counterparts. Furthermore, limit cycle oscillations in the system joint angle outputs, generated by the friction forces, significantly decrease when an on-line compensation system is applied.

Section 2 outlines the theoretical foundations of LPV estimator design and introduces the LMI-based poleplacement concept. In Section 3 , the two-link manipulator case study example is described and the Stribeck approach to modelling the friction forces is outlined prior to constructing the corresponding active LPV FTC scheme. Section 4 provides a concluding discussion of the work.

\section{Theoretical foundation of a robust LPV estimator}

The LPV model can be abstracted as a linear system with state-space matrices that are fixed with some vector of varying parameters (Leith and Leithead, 2000; Wu, 2001). Hence, a nonlinear system is reduced to LPV representation through linearisation along trajectories of the parameters. The nominal LPV system is expressed as

$$
\begin{aligned}
& \dot{x}=A(\theta) x+B(\theta) u, \\
& y=C(\theta) x+D(\theta) u,
\end{aligned}
$$

where $A(\theta) \in \mathbb{R}^{n \times n}, B(\theta) \in \mathbb{R}^{n \times r}, C(\theta) \in \mathbb{R}^{m \times n}$ and $D(\theta) \in \mathbb{R}^{m \times r}$

In contrast to Multiple Model Switching and Tuning (MMST) and Interactive Multiple Model (IMM) methods, LPV controllers or estimators do not require all local linearisation points (Wu, 2001; Leith and Leithead, 2000). The LPV design approach is well posed for a type of nonlinear systems in which the linearisation points cannot be determined or are not unique, e.g., the two-link manipulator system.

The LPV system with faults is described as follows:

$$
\begin{aligned}
& \dot{x}_{p}=A_{p}(\theta) x_{p}+B_{p}(\theta) u+E_{p}(\theta) d+F_{p}(\theta) f, \\
& y_{p}=C_{p}(\theta) x_{p}+D_{p}(\theta) u+G_{p}(\theta) d+H_{p}(\theta) f,
\end{aligned}
$$

where, $x_{p} \in \mathbb{R}^{n}, u \in \mathbb{R}^{r}, y_{p} \in \mathbb{R}^{m}$ and $d \in \mathbb{R}^{q}$ are the states, control inputs, outputs, and disturbances, while $f \in \mathbb{R}^{g}$ is the fault vector. $\theta \in \mathbb{R}^{s}$ is a varying parameter vector, and $A_{p}(\theta), B_{p}(\theta), C_{p}(\theta), D_{p}(\theta), E_{p}(\theta), F_{p}(\theta)$, $G_{p}(\theta)$ and $H_{p}(\theta)$ are matrices with appropriate dimensions.

Based upon the work of Apkarian et al. (1995), the assumptions that apply to fault LPV system (2) include the following:

(A1) The parameter dependence is affine, that is, the statespace matrices $A(\theta), B(\theta), C(\theta), D(\theta), E(\theta), F(\theta)$, $G(\theta)$ and $H(\theta)$ depend affinely on $\theta$. 


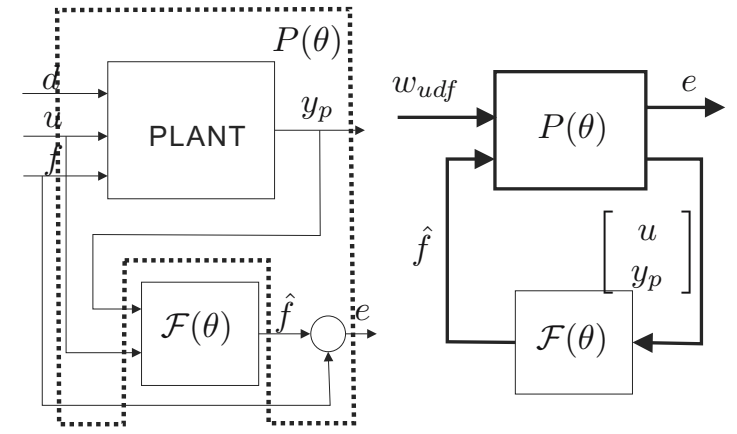

Fig. 1. Polytopic LPV estimation system structure.

(A2) The time-varying parameter $\theta$ varies in a polytope $\Theta$ of vertices $\theta_{1}, \theta_{2}, \ldots, \theta_{j}\left(j=2^{s}\right)$, that is

$$
\begin{aligned}
\theta \in \Theta: & =\operatorname{Co}\left\{\theta_{1}, \theta_{2}, \ldots, \theta_{j}\right\} \\
& =\left\{\sum_{i=1}^{j} a_{i} \theta_{i}: a_{i} \geq 0, \sum_{i=1}^{j} a_{i}=1\right\} .
\end{aligned}
$$

From (A1) and (A2), it is clear that the state-space matrices in (2) range in a polytope of matrices whose vertices are the image of the vertices $\theta_{1}, \theta_{2}, \ldots, \theta_{j}\left(j=2^{s}\right)$. In other words,

$$
\begin{aligned}
& {\left[\begin{array}{llll}
A_{p}(\theta) & B_{p}(\theta) & E_{p}(\theta) & F_{p}(\theta) \\
C_{p}(\theta) & D_{p}(\theta) & G_{p}(\theta) & H_{p}(\theta)
\end{array}\right] \in} \\
& \operatorname{Co}\left\{\left[\begin{array}{llll}
A_{p}\left(\theta_{i}\right) & B_{p}\left(\theta_{i}\right) & E_{p}\left(\theta_{i}\right) & F_{p}\left(\theta_{i}\right) \\
C_{p}\left(\theta_{i}\right) & D_{p}\left(\theta_{i}\right) & G_{p}\left(\theta_{i}\right) & H_{p}\left(\theta_{i}\right)
\end{array}\right]\right\} . \\
&
\end{aligned}
$$

The polytopic estimator $\mathcal{F}(\theta)$ can be written as

$$
\begin{gathered}
\dot{x}_{f}=A_{f}(\theta) x_{f}+B_{f}(\theta)\left[\begin{array}{c}
u \\
y_{p}
\end{array}\right], \\
\widehat{f}=C_{f}(\theta) x_{f}+D_{f}(\theta)\left[\begin{array}{c}
u \\
y_{p}
\end{array}\right],
\end{gathered}
$$

wherein $x_{f} \in \mathbb{R}^{n}$ is the state vector of the estimator, $\hat{f}$ is the estimation of the fault $f$, and $A_{f}(\theta), B_{f}(\theta), C_{f}(\theta)$ and $D_{f}(\theta)$ are matrices with appropriate dimensions to be designed. The LPV estimator (5) can also be rewritten as

$$
\left.\begin{array}{rl}
\mathcal{F}(\theta): & =\left[\begin{array}{cc}
A_{f}(\theta) & B_{f}(\theta) \\
C_{f}(\theta) & D_{f}(\theta)
\end{array}\right] \\
& \in \operatorname{Co}\left\{\left[\begin{array}{cc}
A_{f}\left(\theta_{i}\right) & B_{f}\left(\theta_{i}\right) \\
C_{f}\left(\theta_{i}\right) & D_{f}\left(\theta_{i}\right)
\end{array}\right]\right\} . \\
i=1, \ldots, j
\end{array}\right\} .
$$

The structure of the estimator is shown in Fig. 1. wherein the estimated error vector $e=\hat{f}-f \in \mathbb{R}^{g}$ requires to be minimised. Here $u$ and $y_{p}$ are shown in (2).
Define $x_{p f}$ and $w_{u d f}$ to be $\left[x_{p} x_{f}\right]^{T}$ and $[u d f]^{T}$, respectively. Rewrite (2) as

$$
\begin{aligned}
\dot{x_{p}} & =A x_{p}+B_{1} w_{u d f}+B_{2} \hat{f}, \\
e & =C_{1} x_{p}+D_{11} w_{u d f}+D_{12} \hat{f}, \\
y_{p} & =C_{2} x_{p}+D_{12} w_{u d f}+D_{22} \hat{f},
\end{aligned}
$$

wherein $A=A_{p}, B_{1}=\left[\begin{array}{lll}B_{p} & E_{p} & F_{p}\end{array}\right], C_{2}=0, C_{1}=$ $B_{2}=D_{22}=0, C_{2}=C_{p}, D_{11}=\left[\begin{array}{lll}0 & 0 & -I\end{array}\right], D_{12}=I$, $D_{12}=\left[D_{p} G_{p} H_{p}\right]$.

The above system of Fig. 1 1 can then be expressed as

$$
\begin{aligned}
\dot{x}_{p f} & =A^{\prime}(\theta) x_{p f}+B^{\prime}(\theta) w_{u d f}, \\
e & =C^{\prime}(\theta) x_{p f}+D^{\prime}(\theta) w_{u d f},
\end{aligned}
$$

where

$$
\begin{array}{rlrl}
A^{\prime}(\theta) & =A_{0}+\mathcal{B} \mathcal{F}(\theta) \mathcal{C}, & B^{\prime}(\theta)=B_{0}+\mathcal{B F}(\theta) \mathcal{D}_{21}, \\
C^{\prime}(\theta) & =\mathcal{D}_{12} \mathcal{F}(\theta) \mathcal{C}, & D^{\prime}(\theta)=D_{11}+\mathcal{D}_{12} \mathcal{F}(\theta) \mathcal{D}_{21}, \\
A_{0} & =\left[\begin{array}{cc}
A & 0 \\
0 & 0
\end{array}\right], & B_{0} & =\left[\begin{array}{c}
B_{1} \\
0
\end{array}\right], \\
\mathcal{B} & =\left[\begin{array}{cc}
0 & B_{2} \\
I & 0
\end{array}\right], & \mathcal{C} & =\left[\begin{array}{cc}
0 & I \\
C_{2} & 0
\end{array}\right], \\
\mathcal{D}_{12} & =\left[\begin{array}{ll}
0 & D_{12}
\end{array}\right], & \mathcal{D}_{21} & =\left[\begin{array}{c}
0 \\
D_{21}
\end{array}\right]
\end{array}
$$

The definition of the LMI region and the existence theorem for assignable poles inside this region are given as follows:

Definition 1. (LMI region) A subset $\mathcal{D}$ of the complex plane is called an LMI region if there exist a symmetric matrix $\iota=\left[\iota_{k l}\right]$ and a matrix $\kappa=\left[\kappa_{k l}\right]$ such that

$$
\mathcal{D}=\left\{z \in \mathbf{C}: f_{\mathcal{D}}<0\right\}
$$

with

$$
f_{\mathcal{D}}:=\iota+z \kappa+\bar{z} \kappa^{T}=\left[\iota_{k l}+\kappa_{k l} z+\kappa_{l k} \bar{z}\right]_{l<k, l<m} .
$$

Theorem 1. (Chilali, 1996) The matrix $\Gamma$ is $\mathcal{D}$-stable if and only if there exists a symmetric matrix $H$ such that

$$
T_{\mathcal{D}}(\Gamma, H)<0, \quad H>0
$$

where

$$
\begin{aligned}
T_{\mathcal{D}}(\Gamma, H) & =\iota \otimes H+\kappa \otimes(\Gamma H)+\kappa^{T} \otimes(\Gamma H)^{T} \\
& =\left[\iota_{k l} H+\kappa_{k l} A H+\kappa_{l k} H A^{T}\right]_{l<k, l<m} .
\end{aligned}
$$


Here, Problem 1 is defined to solve the estimation problem with pole-placement.

Problem 1. For the LPV system (2) with the assumptions (A1) and (A2), design a polytopic LPV estimator (5), such that the $L_{2}$-induced norm of the operator mapping $w_{u d f}$ into $e$ is bounded by a scalar number $\gamma$ for all parameter trajectories $\theta$ in the polytope $\Theta$. Also, all poles of the LPV system (10) lie in the given LMI region $\mathcal{D}$. The corresponding sub-optimal $H_{\infty}$ performance is given by

$$
\sup _{0<\left\|w_{u d f}\right\|_{2}<\infty} \frac{\|e\|_{2}}{\left\|w_{u d f}\right\|_{2}}<\gamma
$$

for all $\theta \in \Theta$.

Lemma 1. (Apkarian et al., 1995) For the LPV system (10), the following statements are equivalent:

(i) $\mathrm{L}_{2}$-induced norm of the operator mapping $w_{u d f}$ into $e$ is bounded by a scalar number $\gamma$ for all parameter trajectories $\theta$ in the polytope $\Theta$.

(ii) For the parameter trajectories $\theta$ in the polytope $\Theta$, there exists $P=P^{T}>0$ satisfying the system of LMIs:

$$
\left[\begin{array}{ccc}
X A^{\prime}(\theta)+A^{\prime T}(\theta) P & P B^{\prime}(\theta) & C^{\prime T}(\theta) \\
B^{\prime T}(\theta) P & -\gamma I & D^{\prime T}(\theta) \\
C^{\prime}(\theta) & D^{\prime}(\theta) & -\gamma I
\end{array}\right]<0
$$

where $i=1, \ldots, j$.

The main result of this section is stated in Theorem 2 . which provides the solution of Problem 1.

Theorem 2. Consider the LPV system in (2) with Assumptions $A 1$ and A2. Let $\mathcal{N}_{\mathcal{R}}=\left[\begin{array}{ll}N_{1}^{T} & N_{2}^{T}\end{array}\right]^{T}$ and $\mathcal{N}_{\mathcal{S}}=$ $\left[V_{1}^{T} V_{2}^{T}\right]^{T}$ denote the bases of the null spaces of $\left[B_{2}^{T} D_{12}^{T}\right]$ and $\left[\begin{array}{ll}C_{2} & D_{21}\end{array}\right]$, respectively. There exists a polytopic $L P V$ estimator that can determine the solution of Problem 1 if matrices $0<R=R^{T} \in \mathbb{R}^{n \times n}, 0<S=S^{T} \in \mathbb{R}^{n \times n}$ can be found such that

$$
\begin{aligned}
& {\left[\begin{array}{cc}
\mathcal{N}_{\mathcal{R}} & 0 \\
0 & I
\end{array}\right]^{T}\left[\begin{array}{ccc}
A_{p}\left(\theta_{i}\right) R+R A_{p}^{T}\left(\theta_{i}\right) & 0 & B_{1}\left(\theta_{i}\right) \\
0 & -\gamma I & D_{11} \\
B_{1}^{T}\left(\theta_{i}\right) & D_{11}^{T} & -\gamma I
\end{array}\right]} \\
& {\left[\begin{array}{cc}
\mathcal{N}_{\mathcal{R}} & 0 \\
0 & I
\end{array}\right]<0, \quad i=1, \ldots, j} \\
& {\left[\begin{array}{cc}
\mathcal{N}_{\mathcal{S}} & 0 \\
0 & I
\end{array}\right]^{T}\left[\begin{array}{ccc}
S A_{p}\left(\theta_{i}\right)+A_{p}^{T}\left(\theta_{i}\right) S & S B_{1}\left(\theta_{i}\right) & 0 \\
B_{1}^{T}\left(\theta_{i}\right) S & -\gamma I & D_{11}^{T} \\
0 & D_{11} & -\gamma I
\end{array}\right]} \\
& {\left[\begin{array}{cc}
\mathcal{N}_{\mathcal{S}} & 0 \\
0 & I
\end{array}\right]<0, \quad i=1, \ldots, j,}
\end{aligned}
$$

$$
\begin{array}{r}
U_{1}^{T}\left(\alpha_{k l} S+\beta_{k l} A_{p}\left(\theta_{i}\right) S+\beta_{l k} S A_{p}^{T}\left(\theta_{i}\right)\right) U_{1}<0, \\
i=1, \ldots, j \\
V_{1}^{T}\left(\alpha_{k l} R+\beta_{k l} R A_{p}\left(\theta_{i}\right)+\beta_{l k} A_{p}^{T}\left(\theta_{i}\right) R\right) V_{1}<0, \\
i=1, \ldots, j, \\
{\left[\begin{array}{cc}
R & I \\
I & S
\end{array}\right] \geq 0, \quad i=1, \ldots, j .}
\end{array}
$$

Proof. Based upon Theorem 1, the poles of the LPV system (10) lie in the LMI region $\mathcal{D}$ if and only if there exists $X$ such that

$$
\begin{aligned}
& M_{\mathcal{D}}\left(A^{\prime}(\theta), X\right) \\
& \quad=\left[\alpha_{k l} X+\beta_{k l} A^{\prime}(\theta) X+\beta_{l k} X A^{\prime T}(\theta)\right]<0 .
\end{aligned}
$$

By Lemma 1 Theorem 1 and considering the notation in (5)-(14), there exists a polytopic LPV fault estimator (5) which solves Problem 1 if

$$
\begin{gathered}
\Psi\left(\theta_{i}\right)+U_{x}^{T} \mathcal{F}\left(\theta_{i}\right) V+V^{T} \mathcal{F}^{T}\left(\theta_{i}\right) U_{x}<0, \\
\Phi\left(\theta_{i}\right)+\mathcal{P}^{T} \mathcal{F}\left(\theta_{i}\right) \mathcal{Q}_{\S}+\mathcal{Q}_{\S}^{T} \mathcal{F}^{T}\left(\theta_{i}\right) \mathcal{P}<0,
\end{gathered}
$$

where

$$
\Psi\left(\theta_{i}\right)=\left[\begin{array}{ccc}
X A_{0}\left(\theta_{i}\right)+A_{0}^{T}\left(\theta_{i}\right) X & X B\left(\theta_{i}\right) & 0 \\
B^{T}\left(\theta_{i}\right) X & -\gamma I & D_{11}^{T} \\
0 & D_{11} & -\gamma I
\end{array}\right],
$$

$$
\begin{aligned}
\Phi\left(\theta_{i}\right) & =\alpha_{k l} X+\beta_{k l} A_{0} X+\beta_{l k} X A_{0}^{T}, \\
U_{x} & =\left[\begin{array}{lll}
\mathcal{B}^{T} X & 0 & \mathcal{D}_{12}^{T}
\end{array}\right], \\
V & =\left[\begin{array}{lll}
\mathcal{C} & \mathcal{D}_{21} & 0
\end{array}\right] \\
\mathcal{P} & =\beta_{l k} \mathcal{B}^{T}, \quad \mathcal{Q}_{\S}=\mathcal{C} X .
\end{aligned}
$$

Based on the projection lemma, the LMIs of (23) and (24) hold for some $\mathcal{F}\left(\theta_{i}\right)$ if and only if

$$
\begin{gathered}
W_{U_{x}}^{T} \Psi\left(\theta_{i}\right) W_{U_{x}}<0, \\
W_{V}^{T} \Psi\left(\theta_{i}\right) W_{V}<0, \\
W_{\mathcal{P}}^{T} \Phi\left(\theta_{i}\right) W_{\mathcal{P}}<0, \\
W_{\mathcal{Q}_{\S}}^{T} \Phi\left(\theta_{i}\right) W_{\mathcal{Q}_{\S}}<0,
\end{gathered}
$$

where $W_{U_{x}}, W_{V}, W_{\mathcal{P}}$ and $W_{\mathcal{Q}_{\S}}$ denote any bases of the null spaces of $U_{x}, V, \mathcal{P}$ and $\mathcal{Q}_{\S}$, respectively.

Observing that

$$
\begin{gathered}
U_{x}=\left[\begin{array}{ccc}
\mathcal{B}^{T} & 0 & \mathcal{D}_{12}^{T}
\end{array}\right]\left[\begin{array}{ccc}
X & 0 & 0 \\
0 & I & 0 \\
0 & 0 & I
\end{array}\right], \\
U=\left[\begin{array}{ccc}
\mathcal{B}^{T} & 0 & \mathcal{D}_{12}^{T}
\end{array}\right], \\
\mathcal{Q}=\mathcal{C}
\end{gathered}
$$


the basis for the null space of $U_{x}$ and $\mathcal{Q}_{\S}$ is given by

$$
\begin{gathered}
W_{U_{x}}=\left[\begin{array}{ccc}
X^{-1} & 0 & 0 \\
0 & I & 0 \\
0 & 0 & I
\end{array}\right] W_{U}, \\
W_{\mathcal{Q}_{\S}}=X^{-1} W_{\mathcal{Q}},
\end{gathered}
$$

where $W_{U}$ and $W_{\mathcal{Q}}$ denote any basis of the null space of $U$ and $\mathcal{Q}$. Hence, the inequalities $(30)$ and $(33)$ can be rewritten as

$$
\begin{aligned}
& W_{U}^{T} \Omega\left(\theta_{i}\right) W_{U}<0, \\
& W_{\mathcal{Q}}^{T} \Pi\left(\theta_{i}\right) W_{\mathcal{Q}}<0,
\end{aligned}
$$

with

$$
\Omega\left(\theta_{i}\right)=\left[\begin{array}{ccc}
A_{0}\left(\theta_{i}\right) X^{-1}+X^{-1} \mathbf{A}_{0}^{T}\left(\theta_{i}\right) & B_{0}\left(\theta_{i}\right) & 0 \\
B_{0}^{T}\left(\theta_{i}\right) & -\gamma I & D_{11}^{T} \\
0 & D_{11} & -\gamma I
\end{array}\right],
$$$$
\Pi\left(\theta_{i}\right)=\left(X^{-1}\right)^{T} \alpha_{k l}+\left(X^{-1}\right)^{T} \beta_{k l} A_{0}+\beta_{l k} A_{0}^{T} X^{-1} \text {. }
$$

$X$ and $X^{-1}$ can be partitioned as

$$
X=\left[\begin{array}{cc}
S & N \\
N^{T} & *
\end{array}\right], \quad X^{-1}=\left[\begin{array}{cc}
R & M \\
M^{T} & *
\end{array}\right],
$$

where $S, R, M, N \in \mathbb{R}^{n \times n}$ and $S, R, M>0$, and * stands for the matrix entries which are not of interest.

Then, $X$ and $X^{-1}$ are substituted into (25), 26), (41) and (42), yielding $\Psi\left(\theta_{i}\right), \Phi\left(\theta_{i}\right), \Omega\left(\theta_{i}\right)$ and $\Pi\left(\theta_{i}\right)$ :

$$
\begin{gathered}
\Psi\left(\theta_{i}\right)=\left[\begin{array}{cccc}
S A_{p}\left(\theta_{i}\right)+A_{p}^{T}\left(\theta_{i}\right) S & A_{p}^{T}\left(\theta_{i}\right) N & S B_{1}\left(\theta_{i}\right) & 0 \\
N^{T} A_{p}\left(\theta_{i}\right) & 0 & N^{T} B_{1}\left(\theta_{i}\right) & 0 \\
B_{1}^{T}\left(\theta_{i}\right) & B_{1}^{T}\left(\theta_{i}\right) N & -\gamma I & D_{11}^{T} \\
0 & 0 & D_{11} & -\gamma I
\end{array}\right], \\
\Omega\left(\theta_{i}\right)=\left[\begin{array}{cccc}
A_{p}\left(\theta_{i}\right) R+R A_{p}^{T}\left(\theta_{i}\right) & A_{p}\left(\theta_{i}\right) M & B_{1}\left(\theta_{i}\right) & 0 \\
M^{T} A_{p}^{T}\left(\theta_{i}\right) & 0 & 0 & 0 \\
B_{1}^{T}\left(\theta_{i}\right) & 0 & -\gamma I & D_{11}^{T} \\
0 & 0 & D_{11} & -\gamma I
\end{array}\right],
\end{gathered}
$$

$$
\begin{aligned}
\Phi\left(\theta_{i}\right)=\alpha_{k l} & {\left[\begin{array}{cc}
S & N \\
N^{T} & *
\end{array}\right]+\beta_{k l}\left[\begin{array}{cc}
A_{p}\left(\theta_{i}\right) S & A_{p}\left(\theta_{i}\right) N \\
0 & 0
\end{array}\right] } \\
& +\beta_{l k}\left[\begin{array}{cc}
S A_{p}^{T}\left(\theta_{i}\right) & 0 \\
N^{T} A_{p}^{T}\left(\theta_{i}\right) & 0
\end{array}\right], \\
& i=1, \ldots, j,
\end{aligned}
$$

$$
\begin{aligned}
\Pi\left(\theta_{i}\right)= & \alpha_{k l}\left[\begin{array}{cc}
R & M \\
M^{T} & *
\end{array}\right]+\beta_{k l}\left[\begin{array}{cc}
R A_{p}\left(\theta_{i}\right) & 0 \\
M^{T} A_{p}\left(\theta_{i}\right) & 0
\end{array}\right] \\
& +\beta_{l k}\left[\begin{array}{cc}
A_{p}^{T}\left(\theta_{i}\right) R & A_{p}^{T}\left(\theta_{i}\right) M \\
0 & 0
\end{array}\right] \\
& i=1, \ldots, j .
\end{aligned}
$$

Let

$$
\mathcal{N}_{\mathcal{R}}=\left[\begin{array}{c}
U_{1} \\
U_{2}
\end{array}\right], \quad \mathcal{N}_{\mathcal{S}}=\left[\begin{array}{l}
V_{1} \\
V_{2}
\end{array}\right]
$$

denote the bases of the null spaces of $\left[\begin{array}{ll}B_{2}^{T} & D_{12}^{T}\end{array}\right]$ and $\left[\begin{array}{ll}C_{2} & D_{21}\end{array}\right]$, respectively, where $U_{1}$ and $V_{1}$ span the null spaces of $B_{2}^{T}$ and $C_{2}$. Then, the bases of the null spaces of $U, V, \mathcal{P}$ and $\mathcal{Q}$ are respectively given by

$$
\begin{aligned}
& W_{U}=\left[\begin{array}{cc}
U_{1} & 0 \\
0 & 0 \\
0 & I \\
U_{2} & 0
\end{array}\right], \quad W_{V}=\left[\begin{array}{cc}
V_{1} & 0 \\
0 & 0 \\
0 & I \\
V_{2} & 0
\end{array}\right], \\
& W_{\mathcal{P}}=\left[\begin{array}{cc}
U_{1} & 0 \\
0 & 0
\end{array}\right], \quad W_{\mathcal{Q}}=\left[\begin{array}{cc}
V_{1} & 0 \\
0 & 0
\end{array}\right] .
\end{aligned}
$$

Substituting (44)-(49) into (31), (32), 39) and (40), yields

$$
\begin{aligned}
& {\left[\begin{array}{cc}
\mathcal{N}_{\mathcal{R}} & 0 \\
0 & I
\end{array}\right]^{T}\left[\begin{array}{ccc}
A_{p}\left(\theta_{i}\right) R+R A_{p}^{T}\left(\theta_{i}\right) & 0 & B_{1}\left(\theta_{i}\right) \\
0 & -\gamma I & D_{11} \\
B_{1}^{T}\left(\theta_{i}\right) & D_{11}^{T} & -\gamma I
\end{array}\right]} \\
& {\left[\begin{array}{cc}
\mathcal{N}_{\mathcal{R}} & 0 \\
0 & I
\end{array}\right]<0, \quad i=1, \ldots, j,} \\
& {\left[\begin{array}{cc}
\mathcal{N}_{\mathcal{S}} & 0 \\
0 & I
\end{array}\right]^{T}\left[\begin{array}{ccc}
S A_{p}\left(\theta_{i}\right)+A_{p}^{T}\left(\theta_{i}\right) S & S B_{1}\left(\theta_{i}\right) & 0 \\
B_{1}^{T}\left(\theta_{i}\right) S & -\gamma I & D_{11}^{T} \\
0 & D_{11} & -\gamma I
\end{array}\right]} \\
& {\left[\begin{array}{cc}
\mathcal{N}_{\mathcal{S}} & 0 \\
0 & I
\end{array}\right]<0, \quad i=1, \ldots, j,} \\
& U_{1}^{T}\left(\alpha_{k l} S+\beta_{k l} A_{p}\left(\theta_{i}\right) S+\beta_{l k} S A_{p}^{T}\left(\theta_{i}\right)\right) U_{1}<0, \\
& i=1, \ldots, j \text {, } \\
& V_{1}^{T}\left(\alpha_{k l} R+\beta_{k l} R A_{p}\left(\theta_{i}\right)+\beta_{l k} A_{p}^{T}\left(\theta_{i}\right) R\right) V_{1}<0, \\
& i=1, \ldots, j,
\end{aligned}
$$

Based on the matrix completion result, the condition $X>0$ is equivalent to

$$
\left[\begin{array}{ll}
R & I \\
I & S
\end{array}\right] \geq 0
$$

which completes the proof of Theorem 2

Once the matrices $R$ and $S$ defined in Theorem 2 are obtained, the LPV estimator can be constructed as the following Algorithm 1.

\section{Algorithm 1.}

Step 1: Compute the full rank matrices $M, N$ using SVD such that

$$
M N^{T}=I-R S .
$$


Step 2: Compute $X$ as the unique solution of the linear matrix equation:

$$
X\left[\begin{array}{cc}
I & R \\
0 & M^{T}
\end{array}\right]=\left[\begin{array}{cc}
S & I \\
N^{T} & 0
\end{array}\right]^{T} .
$$

Step 3: Compute $\mathcal{F}\left(\theta_{i}\right)$ by solving (23) and (24).

Step 4: Solve the polytopic LPV estimator:

$$
\mathcal{F}(\theta)=\sum_{i=1}^{j} a_{p}^{i} \mathcal{F}\left(\theta_{i}\right),
$$

where $a_{p}^{i}$ is any solution of the convex decomposition problem

$$
\theta=\sum_{i=1}^{j} a_{p}^{i} \theta_{i}
$$

\section{Two-link manipulator case study}

3.1. Robust LPV fault estimator for a two-link manipulator. A two-link manipulator demonstrator system (shown in Fig. 2) with actuator faults is illustrated. The actuator faults are regarded as friction forces acting at the joints of the system, changing in terms of the angular velocity of the joints. Four types of dynamic torques arise from the motion of the manipulator: inertial, centripetal, coriolis and friction torques. Inertial torques are proportional to the acceleration of each joint in accordance with Newton's second law. Centripetal torques arise from the centripetal forces which constrain a body to rotate around a point. They are directed towards the centre of the uniform circular motion, and are proportional to the square of the velocity. Coriolis torques result from vertical forces derived from the interaction of two rotating links and are proportional to the product of the joint velocities of those links. The model is shown in Fig. 2. The positions of the two arms are defined by the joint angles $\phi=\left[\begin{array}{ll}\phi_{1} & \phi_{2}\end{array}\right]^{T}$. The system inputs are $u=\left[u_{1} u_{2}\right]^{T}$, which are the torques acting onto the joints.

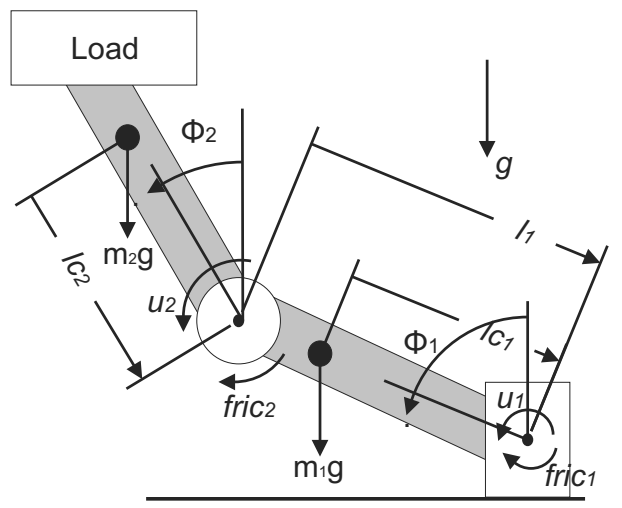

Fig. 2. Two-link manipulator structure.
The dynamics of the manipulator (McKerrow, 1991; Hassen et al., 2000) are shown as

$$
\Xi(\phi) \ddot{\phi}+O(\phi, \dot{\phi}) \dot{\phi}+g(\phi)=u-\operatorname{fric}(\dot{\phi}),
$$

where $\Xi(\phi) \in \mathbb{R}^{2 \times 2}$ is the manipulator inertia tensor matrix, $O(\phi, \dot{\phi}) \dot{\phi} \in \mathbb{R}^{2}$ is the function containing the centripetal and Coriolis torques. $g(\phi) \in \mathbb{R}^{2}$ and fric $(\dot{\phi})$ the gravitational and friction torques, respectively.

The physical system parameters are shown in Table1, with the following notation:

$I_{i}$ : inertia of $\operatorname{arm} i$,

$l_{1}$ : distance between joint 1 and joint 2 ,

$l_{c 1}$ : distance of joint 1 from centre of mass arm 1 ,

$l_{c 2}$ : distance of joint 2 from centre of mass arm 2,

$m_{1}$ : mass of arm 1 and load,

$m_{2}$ : mass of arm 2.

Table 1. Parameter values for the two-link manipulator system.

\begin{tabular}{|c|c|c|}
\hline Parameters & Values [\%] & Units \\
\hline \hline$I_{1}$ & 0.83 & $\mathrm{~kg} \cdot \mathrm{m}^{2}$ \\
\hline$I_{2}$ & 0.41 & $\mathrm{~kg} \cdot \mathrm{m}^{2}$ \\
\hline$l_{1}$ & 1 & $\mathrm{~m}$ \\
\hline$l_{c 1}, l_{c 2}$ & 0.5 & $\mathrm{~kg}$ \\
\hline$m_{1}$ & 10 & $\mathrm{~kg}$ \\
\hline$m_{2}$ & 5 & $\mathrm{~kg}$ \\
\hline$g$ & 9.8 & $\mathrm{~m} / \mathrm{s}^{2}$ \\
\hline
\end{tabular}

The equations of motion including friction on the joints are described by

$$
\begin{gathered}
\left(m_{1} l_{c 1}^{2}+m_{2} l_{1}^{2}+I_{1}\right) \ddot{\phi}_{1}+\left(m_{2} l_{1} l_{c 2} \cos \left(\phi_{1}-\phi_{2}\right)\right) \ddot{\phi}_{2} \\
+m_{2} l_{1} l_{c 2} \sin \left(\phi_{1}-\phi_{2}\right) \dot{\phi}_{2}{ }^{2}-\left(m_{1} l_{c 1}+m_{2} l_{1}\right) g \sin \left(\phi_{1}\right) \\
=u_{1}-\operatorname{fric}_{1}\left(\dot{\phi}_{1}\right), \quad(60) \\
\left(m_{2} l_{1} l_{c 2} \cos \left(\phi_{1}-\phi_{2}\right) \ddot{\phi}_{1}+\left(m_{2} l_{c 2}^{2}+I_{2}\right) \ddot{\phi}_{2}\right. \\
-\left(m_{2} l_{1} l_{c 2} \sin \left(\phi_{1}-\phi_{2}\right)\right) \ddot{\phi}_{1}-m_{2} g l_{c 2} \sin \left(\phi_{2}\right) \\
=u_{2}-\operatorname{fric}_{2}\left(\dot{\phi}_{2}\right) .
\end{gathered}
$$

The friction forces acting on the joints are described by the discontinuous Stribeck friction model (Putra et al., 2004):

$$
f_{\text {fric }}=g\left(x_{p}\right) \operatorname{Sign}\left(x_{p}\right)
$$

where

$$
\operatorname{Sign}\left(x_{p}\right)=\left\{\begin{array}{lll}
-1 & \text { if } & x_{p}<0 \\
{[-1,1]} & \text { if } & x_{p}=0 \\
1 & \text { if } & x_{p}>0
\end{array}\right.
$$


and $g\left(x_{p}\right)=F_{c}+\left(F_{s}-F_{c}\right) \exp \left(-\left|x_{p}\right| / v_{s}\right)^{\delta}$ is the Stribeck friction function with $F_{c}$ and $F_{s}$ being the Coulomb and static friction levels, respectively, and $v_{s}$, $\delta>0$ are the Stribeck velocity and shaping parameters, respectively. In the friction simulation, the following parameter values are used: $F_{c}=5 \mathrm{~N}, v_{s}=0.15 \mathrm{~ms}^{-1}$, $F_{s}=2.5 \mathrm{~N}$ and $\delta_{s}=1.22$. Here, the quadratic terms $O(\phi, \dot{\phi})$ are not considered since they are not bounded. Equation (59) can be simplified as

$$
\Xi(\phi) \ddot{\phi}+g(\phi)=u,
$$

where

$$
\begin{gathered}
\Xi(\phi)=\left[\begin{array}{cc}
m_{1} l_{c 1}^{2}+m_{2} l_{c 1}^{2}+I_{1} & m_{2} l_{1} l_{c 2} \cos \left(\phi_{1}-\phi_{2}\right) \\
m_{2} l_{1} l_{c 2} \cos \left(\phi_{1}-\phi_{2}\right) & m_{2} l_{c 2}^{2}+I_{2}
\end{array}\right], \\
g(\phi)=\left[\begin{array}{c}
-\left(m_{1} l_{c 1}+m_{2} l_{1}\right) g \sin \left(\phi_{1}\right) \\
-m_{2} g l_{c 2} \sin \left(\phi_{2}\right)
\end{array}\right] .
\end{gathered}
$$

The nonlinear term in $\Xi(\phi)$ is clearly a bounded function,

$$
\varphi_{1}(\phi)=\cos \left(\phi_{1}-\phi_{2}\right) \in\left[\begin{array}{ll}
-1 & 1
\end{array}\right] .
$$

Hence, $\Xi(\phi)$ can be represented by a polytope whose vertices are defined by

$$
\Xi(\phi) \in \operatorname{Co}\left\{\Xi_{1} \Xi_{2}\right\}
$$

where

$$
\begin{aligned}
& \Xi_{1}(\phi)=\left[\begin{array}{cc}
m_{1} l_{c 1}^{2}+m_{2} l_{c 1}^{2}+I_{1} & m_{2} l_{1} l_{c 2} \\
m_{2} l_{1} l_{c 2} & m_{2} l_{c 2}^{2}+I_{2}
\end{array}\right], \\
& \Xi_{2}(\phi)=\left[\begin{array}{cc}
m_{1} l_{c 1}^{2}+m_{2} l_{c 1}^{2}+I_{1} & -m_{2} l_{1} l_{c 2} \\
-m_{2} l_{1} l_{c 2} & m_{2} l_{c 2}^{2}+I_{2}
\end{array}\right] .
\end{aligned}
$$

To construct a state-space formulation, the vector field $g(\phi)$ with $\varphi \in \mathbb{R}^{2}$ can be arranged in the form of $G^{g}(\phi) \phi$ and the function $\varphi_{2}(\phi)$ can now be defined, which is bounded:

$$
\begin{aligned}
& \sin \left(\phi_{1}\right)=\left(\frac{\sin \left(\phi_{1}\right)}{\phi_{1}}\right) \phi_{1}=\varphi_{2}\left(\phi_{1}\right) \phi_{1}, \\
& \sin \left(\phi_{2}\right)=\left(\frac{\sin \left(\phi_{2}\right)}{\phi_{2}}\right) \phi_{2}=\varphi_{3}\left(\phi_{2}\right) \phi_{2},
\end{aligned}
$$

where $-0.2 \leq \varphi_{2} \leq 1,-0.2 \leq \varphi_{3} \leq 1$.

From the bound of function $\varphi_{2}\left(\phi_{1}\right), \varphi_{3}\left(\phi_{2}\right)$ in terms of the angle $\phi, G^{g}(\phi)$ is considered to be a polytope as follows:

$$
G^{g}(\phi) \in \operatorname{Co}\left\{G_{1}^{g}, G_{2}^{g}, G_{3}^{g}, G_{4}^{g}\right\}
$$

where

$$
G_{1}^{g}=\left[\begin{array}{cc}
0.2\left(m_{1} l_{c 1}+m_{2} l_{1}\right) g & 0 \\
0 & 0.2 m_{2} l_{c 2} g
\end{array}\right],
$$

$$
\begin{gathered}
G_{2}^{g}=\left[\begin{array}{cc}
-\left(m_{1} l_{c 1}+m_{2} l_{1}\right) g & 0 \\
0 & 0.2 m_{2} l_{c 2} g
\end{array}\right], \\
G_{3}^{g}=\left[\begin{array}{cc}
0.2\left(m_{1} l_{c 1}+m_{2} l_{1}\right) g & 0 \\
0 & -0.2 m_{2} l_{c 2} g
\end{array}\right], \\
G_{4}^{g}=\left[\begin{array}{cc}
-\left(m_{1} l_{c 1}+m_{2} l_{1}\right) g & 0 \\
0 & -m_{2} l_{c 2} g
\end{array}\right] .
\end{gathered}
$$

To define the state space representation of the twolink system, let

$$
x=\left[\begin{array}{llll}
\phi_{1} & \phi_{2} & \dot{\phi}_{1} & \dot{\phi}_{2}
\end{array}\right]^{\prime}
$$

and

$$
W_{b}=\left[\begin{array}{llll}
0 & 0 & 0 & 1 \\
0 & 0 & 1 & 0
\end{array}\right]^{\prime}
$$

The LMI constraints with state feedback according to the nonlinear equations in (59) and (60) are then given by the following descriptor system:

$$
\left[\begin{array}{cc}
I & 0 \\
0 & \Xi(\phi)
\end{array}\right] \dot{x}=\left[\begin{array}{cc}
0 & I \\
-G^{g}(\phi) & 0
\end{array}\right] x+W_{b} u .
$$

Let $\Pi$ be a nonsingular matrix given by

$$
\Pi=\left[\begin{array}{cc}
I & 0 \\
0 & \Xi(\phi)
\end{array}\right]
$$

The state space equation can be expressed as follows:

$$
\dot{x}=A(\phi) x+B(\phi) u,
$$

where

$$
\begin{gathered}
A(\phi)=\Pi^{-1}\left[\begin{array}{cc}
0 & I \\
-G^{g}(\phi) & 0
\end{array}\right], \\
B(\phi)=\Pi^{-1} W_{b} .
\end{gathered}
$$

With the faults, choosing the scheduling parameter as $\theta=\left[\begin{array}{lll}\varphi_{1} & \varphi_{2} & \varphi_{3}\end{array}\right]^{\prime}$,

$$
\begin{aligned}
\dot{x} & =A(\theta) x+B(\theta) u+F_{a} f_{a} \\
& =A_{i j} x+B_{i} u+F_{a} f_{a},
\end{aligned}
$$

$i=1,2$ and $j=1,2,3,4$, where $F_{a}$ is a fault distribution matrix and $f_{a}$ stands for actuator faults which represent the friction forces acting on each joint.

A common constant gain matrix $K$ is built to stabilise the fault-free open-loop system on each vertex. Let $L_{c}=$ $K S_{c}$. Then the quadratic stability conditions for an eightvertex system can be written as

$$
\begin{aligned}
& A_{i j} S_{c}+B_{i} L_{c}+S_{c} A_{i j}^{T}+L_{c}^{T} B_{i}^{T} \leq 0 \\
& \quad S_{c}=S_{c}^{T} \geq I, \quad i=1,2, \quad j=1,2,3,4 .
\end{aligned}
$$


The following matrices $S_{c}$ and $L_{c}$ were calculated using the MATLAB LMI Toolbox after 55 iterations. The corresponding controller is

$$
\begin{aligned}
S_{c} & =\left[\begin{array}{cccc}
2.1051 & 0.0000 & -4.7048 & -0.0000 \\
0.0000 & 2.1396 & -0.0000 & -4.1781 \\
-4.7048 & -0.0000 & 21.0304 & 0.0000 \\
-0.0000 & -4.1781 & 0.0000 & 16.3180
\end{array}\right], \\
L_{c} & =\left[\begin{array}{cccc}
-75.6297 & 0.0000 & -176.2102 & -0.0000 \\
-0.0000 & -20.9682 & 0.0000 & -139.6002
\end{array}\right], \\
K & =\left[\begin{array}{cccc}
-109.3065 & 0 & -32.8321 & 0 \\
0 & -53.0095 & 0 & -22.1276
\end{array}\right] .
\end{aligned}
$$

The eight-vertex of a closed-loop system is combined by $A_{i j}$ and $B_{i}$, which are given as follows:

$$
\begin{aligned}
& A_{11}=\left[\begin{array}{cccc}
0 & 0 & 1.0000 & 0 \\
0 & 0 & 0 & 1.0000 \\
-4.2519 & 9.3277 & -7.1627 & 7.2397 \\
6.3765 & -31.0910 & 10.7419 & -24.1312
\end{array}\right] \text {, } \\
& A_{12}=\left[\begin{array}{cccc}
0 & 0 & 1.0000 & 0 \\
0 & 0 & 0 & 1.0000 \\
-4.2519 & 18.9467 & -7.1627 & 7.2397 \\
6.3765 & -63.1531 & 10.7419 & -24.1312
\end{array}\right] \\
& A_{13}=\left[\begin{array}{cccc}
0 & 0 & 1.0000 & 0 \\
0 & 0 & 0 & 1.0000 \\
-27.7655 & 9.3277 & -7.1627 & 7.2397 \\
41.6399 & -31.0910 & 10.7419 & -24.1312
\end{array}\right] \\
& A_{14}=\left[\begin{array}{cccc}
0 & 0 & 1.0000 & 0 \\
0 & 0 & 0 & 1.0000 \\
-27.7655 & 18.9467 & -7.1627 & 7.2397 \\
41.6399 & -63.1531 & 10.7419 & -24.1312
\end{array}\right] \\
& A_{21}=\left[\begin{array}{cccc}
0 & 0 & 1.0000 & 0 \\
0 & 0 & 0 & 1.0000 \\
-4.2519 & -9.3277 & -7.1627 & -7.2397 \\
-6.3765 & -31.0910 & -10.7419 & -24.1312
\end{array}\right] \text {, } \\
& A_{22}=\left[\begin{array}{cccc}
0 & 0 & 1.0000 & 0 \\
0 & 0 & 0 & 1.0000 \\
-4.2519 & -18.9467 & -7.1627 & -7.2397 \\
-6.3765 & -63.1531 & -10.7419 & -24.1312
\end{array}\right] \text {, } \\
& A_{23}=\left[\begin{array}{cccc}
0 & 0 & 1.0000 & 0 \\
0 & 0 & 0 & 1.0000 \\
-27.7655 & -9.3277 & -7.1627 & -7.2397 \\
-41.6399 & -31.0910 & -10.7419 & -24.1312
\end{array}\right]
\end{aligned}
$$

$$
A_{24}=\left[\begin{array}{cccc}
0 & 0 & 1.0000 & 0 \\
0 & 0 & 0 & 1.0000 \\
-27.7655 & -18.9467 & -7.1627 & -7.2397 \\
-41.6399 & -63.1531 & -10.7419 & -24.1312
\end{array}\right],
$$

$$
\begin{gathered}
B_{1}=\left[\begin{array}{cc}
0 & 0 \\
0 & 0 \\
0.2182 & -0.3272 \\
-0.3272 & 1.0905
\end{array}\right], \\
B_{2}=\left[\begin{array}{cc}
0 & 0 \\
0 & 0 \\
0.2182 & 0.3272 \\
0.3272 & 1.0905
\end{array}\right] .
\end{gathered}
$$

The actuator fault estimate $\hat{f}_{a}$ for system (78) can then be implemented by using Algorithm 1.

The fault-free system outputs of two joint rotation angles can be shown in Figs. 3 and 4

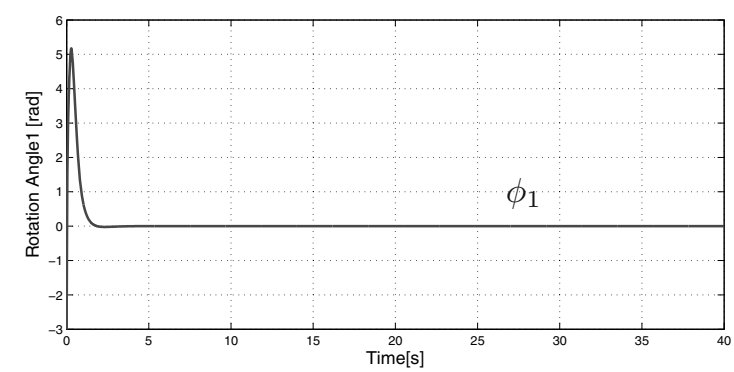

Fig. 3. Fault-free response of Angle 1.

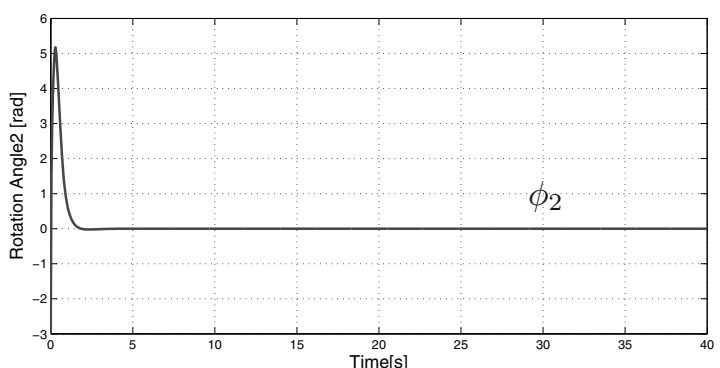

Fig. 4. Fault-free response of Angle 2.

With the faults (friction forces), the nonlinear system outputs of two angles are shown in Figs. 5 and 6, respectively. The corresponding faults (friction forces) are shown in Fig.7

The desired LMI region $\mathcal{D}$ is forced to lie in the halfplane $x<-0.5$. Also, choosing $\gamma$ to be 2.7550 , after 15 iterations, the $\gamma$-suboptimum estimator $\mathcal{F}_{i}(\theta)$ is calculated, corresponding to each vertex. The LPV estimator is then built through the combination of $\mathcal{F}_{i}(\theta), i=$ 


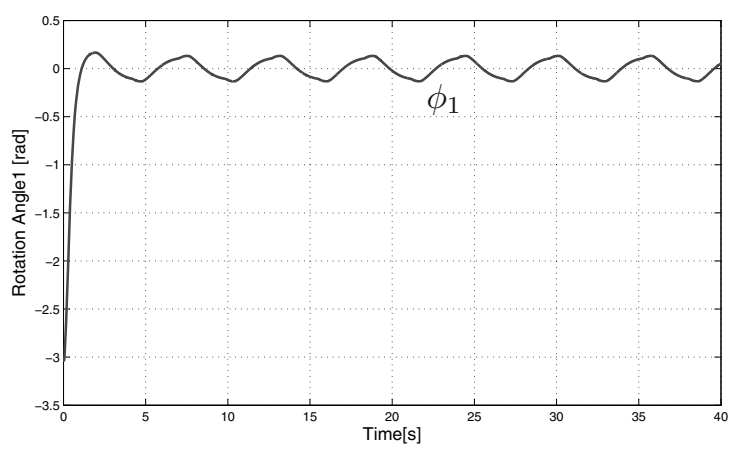

Fig. 5. Response of Angle 1 with faults.

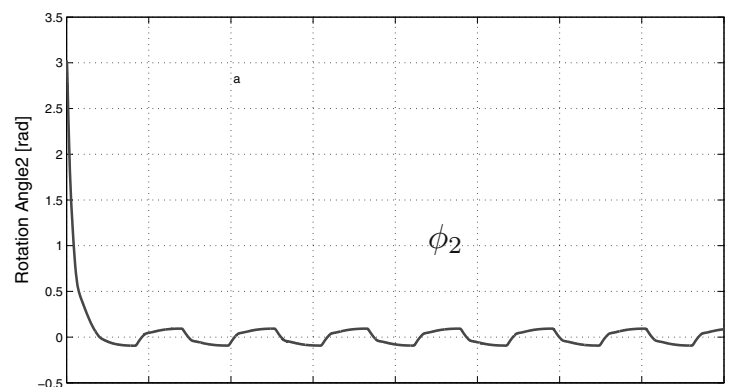

Fig. 6. Response of Angle 2 with faults.
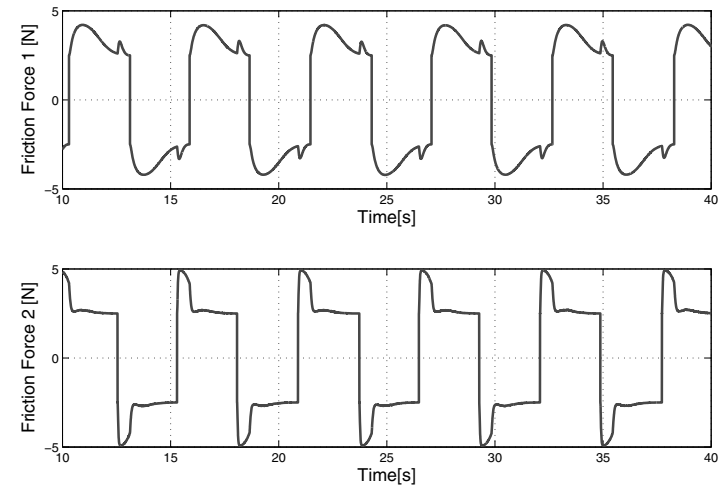

Fig. 7. Friction forces acting on two joints.

$1,2, \ldots, 8$. Figures 8 and 9 show the results of the friction estimation, with a Gaussian random disturbance $d$ of zero-mean and variance 0.02 .

Simulation results show that the robust LPV fault estimator provides a good online estimation performance for both friction forces simultaneously. The solid line represents the fault signal and the dotted line depicts the estimated fault signal.

3.2. Active LPV FTC scheme. The dynamic system of (78) includes an additive term of the actuator faults.

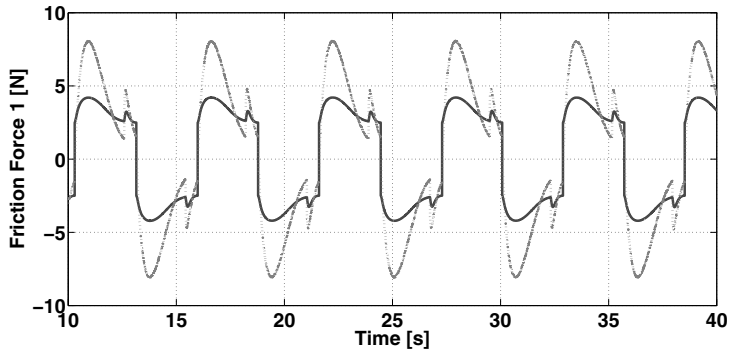

Fig. 8. Estimated friction acting on Joint 1.

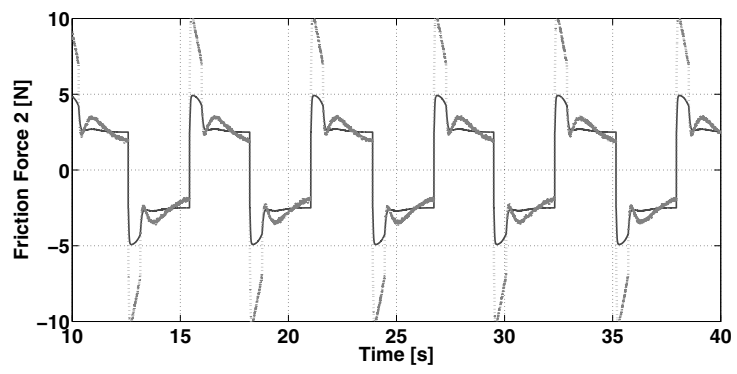

Fig. 9. Estimated friction acting on Joint 2.

However, the faults can have a multiplicative effect in the system representation. A multiplicative actuator fault representation can be defined as

$$
\dot{x}=A_{i j} x+B_{i}\left[I_{r}-\eta^{a}\right] u,
$$

where $\eta^{a}$ is the so-called fault-effect factor, and $\eta^{a}=$ $\operatorname{diag}\left[\eta_{1}^{a}, \eta_{2}^{a}, \ldots, \eta_{r}^{a}\right]$, and $0 \leq \eta_{i}^{a}<1$ represents a fault in the $i$-th actuator while $\eta_{i}^{a}=0$ means that the $i$-th actuator operates normally (fault-free), whilst $\eta_{i}^{a}>0$ means that some degree of the fault effect occurs in the actuator (Chen et al., 1999). The distribution matrix $F_{a}$ is equal to the matrix $B$ in an actuator fault case. The estimation of the fault-effect factor $\eta^{a}$ is determined from the fault estimation $\hat{f}$ provided by the LPV fault estimator.

In Section 3.1, a constant controller is developed to achieve the stability of the eight-vertex system. However, sometimes, specified nonlinear system outputs have to be considered, e.g., the mode distribution or pole position, which require an LPV controller $K_{l p v}$ to be achieved. The LPV controller can be expressed by

$$
K_{l p v}=K+K(\theta)
$$

where $K$ is the developed constant controller.

The structure of the active LPV FTC system is shown in Fig. 10, wherein $K_{\mathrm{FTC}}\left(\hat{\eta}^{a}, \theta\right)$ is the adaptive LPV controller for the FTC mechanism, depending on the on-line estimation $\hat{\eta}^{a}$ and measurement $\theta$.

Theorem 3. Consider the system in (93) with $i=1, \ldots, r$ actuator faults $\left(\eta^{a} \neq 0\right)$ acting independently within the 


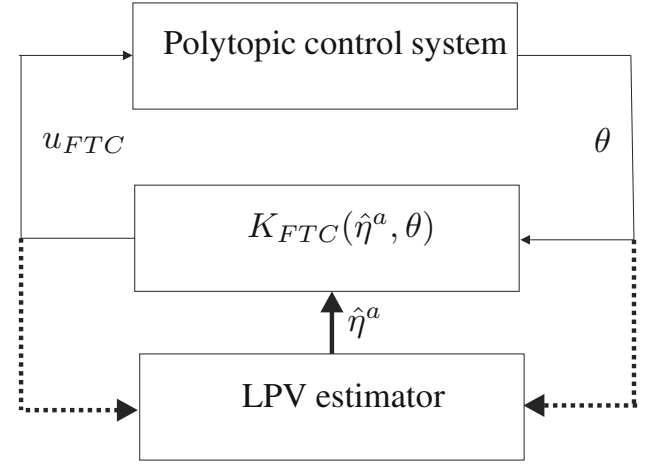

Fig. 10. Active LPV fault-tolerant control scheme.

control system with an LPV gain matrix $K_{l p v}$. Define $\zeta=$ $\left[I-\hat{\eta}^{a}\right]$. The new control action (assuming nonzero fault effects) is given as

$$
\begin{aligned}
u_{\mathrm{FTC}} & =\left(\zeta^{\dagger}+\left(I-\zeta^{\dagger} \zeta\right) Z\right) K_{l p v} x \\
& =K_{\mathrm{FTC}}\left(\hat{\eta}^{a}, \theta\right) x,
\end{aligned}
$$

where $\zeta$ is required to be of full row rank, $\dagger$ represents the pseudo-inverse, $Z$ is the free matrix. The degree of freedom of designing the LPV controller can be fully utilised by choosing various $Z$ (Chen, 2009).

Proof. Based upon the system state equation with faults (93) and the new control input $u_{\mathrm{FTC}}$, the fault compensation system is given by

$$
\begin{aligned}
\dot{x} & =A x+B \zeta u_{\mathrm{FTC}} \\
& =A x+B \zeta\left(\zeta^{\dagger}+\left(I-\zeta^{\dagger} \zeta\right) Z\right) K_{l p v} x \\
& =A x+B u_{\mathrm{nom}} .
\end{aligned}
$$

It can be seen that the term $\left(I-\eta^{a}\right)$ acting on the system (93) can be removed through replacing $u$ with $u_{\mathrm{FTC}}$, which completes the proof.

Figure 12 shows the system outputs after the fault has been compensated. Apparently, the system output oscillations caused by frictions can be decreased. The nominal performance of the system outputs generated by the LPV controller can be effectively recovered after the fault compensation is activated at $40 \mathrm{~s}$. This demonstrates very well the fault-tolerance of the active LPV FTC system.

\section{Conclusions}

The principle of the LPV design approach for robust fault estimation with pole-placement has been introduced through the use of a set of LMIs using efficient interiorpoint algorithms. The robust LPV fault estimator design approach is well posed for fault detection and diagnosis of a nonlinear system. Not only can the robustness of the estimation error be improved, corresponding to the system control inputs, disturbances and the faults, but also

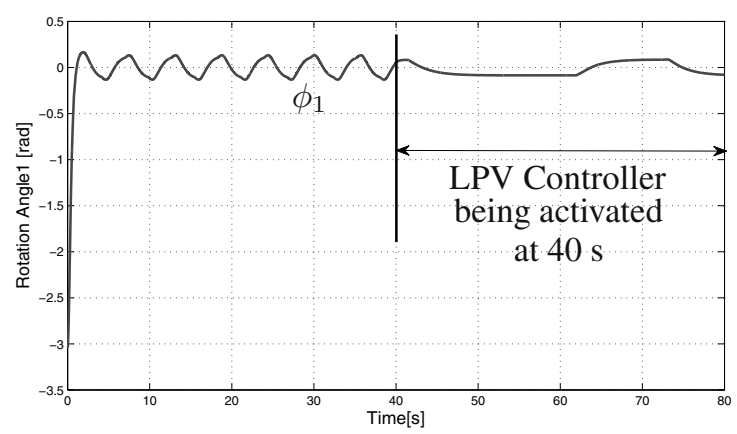

Fig. 11. Angle response of Joint 1 with active FTC.

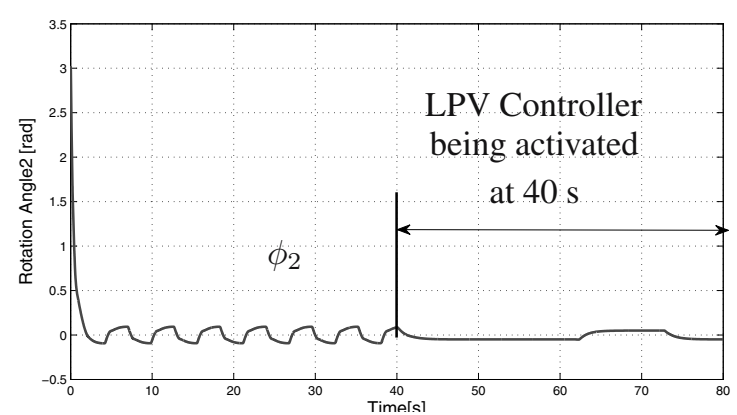

Fig. 12. Angle response of Joint 2 with active FTC.

the structure of the robust LPV estimator can be modified online through the measurement of the varying parameters. As an example of a practical type of actuator fault, the friction forces acting in the joints of a two-joint manipulator system are generated using the Stribeck friction model. The simulation results show that the simultaneously acting friction forces can be estimated effectively using the polytope estimator, and the actuator faults can be compensated through the developed active LPV FTC scheme.

\section{Acknowledgment}

The authors wish to thank the European Commission for research funding in the contract FP7-233815, Advanced Fault Diagnosis for Safer Flight Guidance and Control (ADDSAFE).

\section{References}

Apkarian, P., Gahinet, P. and Becker, G. (1995). Self-scheduled $\mathrm{H}_{\infty}$ control of linear parameter-varying systems: A design example, Automatica 31(9): 1251-1261.

Armstrong-Hélouvry, B., Dupont, P. and de Wit, C.C. (1994). A survey of models, analysis tools and compensation methods for the control of machines with friction, Automatica 30(7): 1083-1138. 
Bokor, J. and Balas, G. (2004). Detection filter design for LPV systems: A geometric approach, Automatica 40(3): 511518 .

Bona, B. and Indri, M. (2005). Friction compensation in robotics: An overview, Proceedings of the 44th IEEE Conference on Decision and Control/European Control Conference 2005, Seville, Spain, pp. 4360-4367.

Casavola, A., Famularo, D., Franze, G. and Patton, R. (2008). A fault detection filter design method for a class of linear time-varying systems, 16th Mediterranean Conference on Control and Automation, Ajaccio, France, pp. 1681-1686.

Casavola, A., Famularo, D., Franze, G. and Sorbara, M. (2007). A fault-detection, filter-design method for linear parameter-varying systems, Proceedings of the Institution of Mechanical Engineers, Part I: Journal of Systems and Control Engineering 221(6): 865-874.

Chen, J., Patton, R. and Chen, Z. (1999). Active fault-tolerant flight control systems design using the linear matrix inequality method, Transactions of Institute of Measurement \& Control 21(2-3): 77-84.

Chen, L. (2009). Multirate Eigenstructure Assignment Using Lifting, Ph.D. thesis, University of York, York.

Ganguli, S., Marcos, A. and Balas, G. (2002). Reconfigurable LPV control design for B-747-100/200 longitudinal axis, American Control Conference, Anchorage, AK, USA, pp. 3612-3617.

Hassen, S., Crusca, F. and Abachi, H. (2000). Modelling system for control studies-An overview, ISCA 15th International Conference on Computers and Their Application, Orlando, FL, USA, pp. 418-421.

Henry, D. and Zolghadri, A. (2005). Design and analysis of robust residual generators for systems under feedback control, Automatica 41(2): 251-264.

Hou, M. and Patton, R. (1996). An LMI approach to $\mathrm{H}_{-} / \mathrm{H}_{\infty}$ fault detection observers, UKACC International Conference on CONTROL '96, Exeter, UK, pp. 305-310.

Leith, D. and Leithead, W. (2000). Survey of gain-scheduling analysis and design, International Journal of Control 73(11): 1001-1025.

Marcos, A., Ganguli, S. and Balas, G. (2005). An application of $\mathrm{H}_{\infty}$ fault detection and isolation to a transport aircraft, Control Engineering Practice 13(1): 105-119.

McKerrow, P. (1991). Introduction to Robotics, Addison-Wesley Publishing Company, Inc., Boston, MA.

Olsson, H., Astrom, K., Wit, C.C.D., Gafvert, M. and Lischinsky, M. (1998). Friction models and friction compensation, European Journal of Control 4(3): 176-195.

Patton, R. and Klinkhieo, S. (2010). LPV fault estimation and FTC of a two-link manipulator, 2010 American Control Conference, Baltimore, MD, USA, pp. 4647-4652.
Patton, R., Putra, D. and Klinkhieo, S. (2010). Friction compensation as fault-tolerant control problem, International Journal of Systems Science 41(8): 987-1001.

Putra, D., Moreau, L. and Nijmeijer, H. (2004). Observer-based compensation of discontinuous friction, Proceedings of the 43rd IEEE Conference on Decision and Control, Atlantis, Paradise Island, The Bahamas, pp. 4940-4945.

Weng, Z., Patton, R. and Cui, P. (2008). Robust fault estimation for linear parameter-varying time-delay systems, 16th Mediterranean Conference on Control and Automation, Ajaccio, France, pp. 292-296.

Wu, F. (2001). A generalised LPV system analysis and control synthesis framework, International Journal of Control 74(7): 745-749.

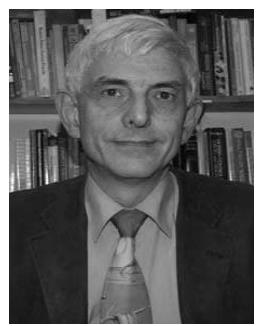

Ron J. Patton (B.Eng., M.Eng., Ph.D., F.Ints.M.C., AIAA Senior Member, IEEE Fellow) is currently a professor of control and intelligent systems engineering at the University of Hull. He was born in Peru in 1949 and was educated at Emmanuel Grammar School, Swansea, graduating from Sheffield University in electrical and electronic engineering in 1972. He has held appointments at the BBC Research Department and at the Royal Free Hospital, London, subsequent to Ph.D. research at Sheffield University in bio-control systems. He was a lecturer and a senior lecturer in control systems at York University (1981-2004), following a period of employment as a lecturer at Sheffield City Polytechnic (now Sheffield Hallam University) (19781981), where he contributed to fundamental work on ship positioning systems based on Kalman filtering. His research currently focuses on robust methods for fault diagnosis and fault-tolerant control for process as well as aerospace and offshore wind turbine systems. He his particularly interested in non-linear system approaches for control and estimation and has substantially contributed to the topic of soft computing for fault diagnosis. He has authored more than 400 publications, including 103 journal papers, 4 books, 3 edited books and 26 book chapters, mainly on fault diagnosis, fault tolerant control and eigenstructure assignment design. He has served on editorial boards of various journals and has been active in the IFAC and IEEE, becoming an IEEE fellow in 2009 for his research work on fault diagnosis and fault-tolerant control. He has also served for 25 years on numerous international conference and symposia committees of the EUCA, IEE, IEEE, IFAC and UKACC

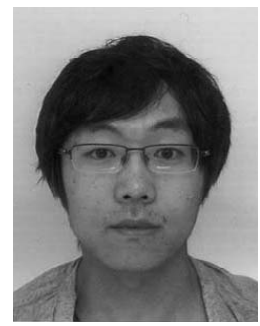

Lejun Chen (B.Eng., Ph.D.) was born in China in 1983. He obtained his Ph.D. degree in electronic engineering from the University of York, UK, in 2009 for research on combining sampleddata theory with eigenstructure assignment to achieve better control robustness and performance for aircraft and practical lab demonstration systems. He is now a postdoctoral research associate at the Control \& Intelligent Systems Engineering (C\&ISE) research group, University of Hull, UK. He is working for the EU-FP7 funded research project on Advanced Fault Diagnosis for Sustainable Flight Guidance and Control (ADDSAFE). His current research interests include nonlinear system fault detection and diagnosis, fault tolerant control for nonlinear systems, sampled-data control, distributed system control, and nonlinear system modeling and control. 


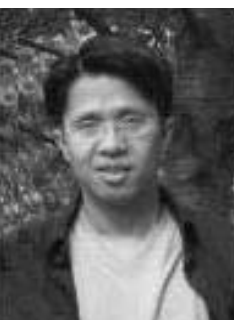

Supat Klinkhieo (B.Sc., M.Sc., Ph.D.) was born in Thailand in 1976. He obtained his B.Sc. degree in telecommunications engineering at the Suranaree University of Technology, Thailand, in 1998, and the M.Sc. in electronic engineering at Hull University, UK, in 2006. In 2010 he was awarded his Ph.D. at Hull University for research on on-line estimation approaches to fault-tolerant control of uncertain systems. His research interests include networked control systems, robust fault diagnosis and fault-tolerant control. He currently works as the head of the Control Department at the Thailand Synchrotron Agency.

Received: 26 January 2011

Revised: 14 September 2011 